\title{
ESPACIOS PÚBLICOS Y GÉNERO EN CENTROS HISTÓRICOS: LAS ALAMEDAS DE SEVILLA Y SANTA CRUZ DE TENERIFE*
}

\author{
Luz Marina García Herrera', Ma del Carmen Díaz Rodríguez', \\ Antonio García García ${ }^{2}$ y Alejandro Armas Díaz ${ }^{1}$ \\ ${ }^{1}$ Departamento de Geografía. Universidad de La Laguna \\ Imgarcía@ull.es, cdiazrod@ull.es, aarmas@ull.es \\ ${ }^{2}$ Departamento de Geografía, Historia y Filosofía. Universidad Pablo de Olavide (Sevilla) \\ agargar1@upo.es
}

\section{RESUMEN}

El artículo estudia dos espacios públicos en los centros históricos de las ciudades de Sevilla y de Santa Cruz de Tenerife: las alamedas de Hércules y del Duque de Santa Elena, respectivamente. Mediante el uso de observaciones directas y entrevistas se exploran los efectos de la reciente reordenación de ambos lugares sobre las dinámicas sociales. Tras un análisis comparado del uso y apropiación de tales espacios, se centra la atención en el reconocimiento de las diferencias existentes entre hombres y mujeres en su uso, poniendo de manifiesto la relevancia de las categorías de género y edad.

Palabras clave: Espacios públicos, género, Sevilla, Santa Cruz de Tenerife.

\section{ABSTRACT}

The article studies two public spaces in the historical centers of Seville and Santa Cruz de Tenerife: the alamedas of Hercules and the Duque de Santa Elena. The effects of their recent redevelopment upon social dynamics are explored through direct observations and

Fecha de recepción: julio 2012.

Fecha de aceptación: julio 2013.

* El trabajo forma parte del Proyecto de Investigación «Ciudad y calidad de vida. El uso social de los espacios públicos abiertos en ciudades españolas» (CSO 2010-19007) financiado por el Plan Nacional de I+D+i del Ministerio de Ciencia e Innovación. 
interviews. While taking into account the similarities and differences in the social practices registered in both public spaces, the aim is to identify gender differences related to public space use. In doing so, this paper shed light on the relevance of categories as gender and age.

Key words: Public spaces, gender, Seville, Santa Cruz de Tenerife.

\section{INTRODUCCIÓN}

La presencia de espacios públicos de calidad en la ciudad es una dimensión clave desde el punto de vista de los derechos ciudadanos, valores universales que se expresan y se desarrollan en cada territorio. Por tanto, ofrecer lugares con cualidades formales adecuadas para incluir a todos, sin excluir a nadie, supone reconocer, en el marco urbano como en tantos otros, la existencia de distintos requerimientos por parte de las personas en el intento cotidiano de ejercer tales derechos. Si se desea favorecer la equidad en la convivencia social es evidente que el planeamiento y la gestión tienen consecuencias relevantes en la vida diaria de las personas y de un modo especial entre los grupos o colectivos, como el de las mujeres, menos favorecidos; de ahí que la reivindicación de una organización no sexista de dichos espacios sea una de las nuevas demandas sociales.

Este artículo aborda, desde la perspectiva de género, el análisis del espacio público haciendo visibles las razones que pueden determinar las diferencias de uso entre hombres y mujeres (Ortiz, 2007: 13), así como las acciones que contribuirían a una mayor igualdad en términos de presencia y participación. El análisis se centra, por tanto, en las diferencias y semejanzas en el uso y apropiación de dos lugares históricos y simbólicos para los habitantes de las ciudades de Sevilla y Santa Cruz de Tenerife, tras las diversas intervenciones físicas que han afectado a sus funciones y dinámicas sociales: las alamedas de Hércules y del Duque de Santa Elena, respectivamente. Se trata de recintos en los que se llevan a cabo transformaciones de gran envergadura que se justifican por la necesidad de revitalizar áreas o sectores urbanos que han perdido dinamismo, al tiempo que se argumenta que de ese modo se logra mejorar la calidad de vida de los residentes. Esta apelación al bienestar individual y colectivo sirve de fundamento para la actuación y a la vez la dota de legitimación y consenso social. Sin embargo, la reordenación o creación de los nuevos espacios colectivos que se derivan de esas acciones, no siempre garantiza un mejor derecho de uso y mayores oportunidades de apropiación no excluyentes. Por tanto, el análisis comparado trata de mostrar que, más allá de las diferencias propias de los contextos locales, la acción de renovación o de producción de nuevos espacios de relación por parte de la administración, si bien favorece la diversificación de los usuarios, no parece, en cambio, como se ha demostrado en otras investigaciones (Paravicini, 2003), que consiga el desarrollo de dinámicas de uso y apropiación más inclusivas.

Mediante una metodología cualitativa, basada en la observación directa y en entrevistas a los usuarios, se examina el significado ciudadano actual de los espacios analizados (Prats, Baylina y Ortiz 2012; García Ramon, Ortiz y Prats 2004; Ortiz 2004). La fuente principal de información, además del obligado análisis documental y bibliográfico, ha sido, por tanto, el reconocimiento y cómputo sistemático, en diferentes días (laborables y festivos) y horas (mañanas y tardes), del número de usuarios, de sus características y de las actividades que éstos desarrollaban en los dos espacios considerados. En la alameda de Hércules los regis- 
tros ascienden a 1.688 y en la de Santa Cruz a 401; valores que se consideran adecuados, atendiendo a las escalas y poblaciones de ambas ciudades, para ofrecer una aproximación sobre el perfil de sus usuarios, así como sobre las pautas de ocupación habituales en ambos lugares. En cada una de las observaciones se procedió a la representación, sobre un croquis, de la localización de los distintos grupos que ocupaban el espacio en cuestión a la vez que se obtenían fotografías y se anotaba en una ficha los rasgos personales y las prácticas que se realizaban de forma individual o grupal. La explotación de esta información aporta resultados cuantitativos - estadísticos, gráficos y cartográficos- sobre los usuarios y usuarias, atendiendo a su edad y a las formas de apropiación.

Los datos básicos, aunque fundamentales, derivados del trabajo de observación se complementan con las entrevistas realizadas mediante cuestionarios abiertos, cuya finalidad era averiguar las razones que llevaban al uso y a la frecuentación del espacio, las vivencias que en él tenían lugar, así como otras características relativas a la frecuencia de uso, a la residencia de los usuarios y los medios de traslado empleados para llegar hasta el lugar. A estos cuestionarios respondieron 40 personas ( 20 en cada alameda) con un peso similar de mujeres y varones de diferentes edades (entre 15 y 82 años). Se trata de una muestra que no tiene por objeto una representatividad estadística, pero sí sustantiva o significativa de las motivaciones que conducen a la estancia en los espacios considerados y las valoraciones que de ellos realizan vecinos y visitantes.

Con la finalidad de conocer el acceso y disfrute ciudadano que se produce en las alamedas bajo estudio, se examinará de forma comparada, por un lado, quiénes, cuándo y cómo las usan y, por otro, se intentará reconocer las variables que inciden en las maneras de estar y compartir según género, en lo que influirá, sin duda, el diferente papel social que de modo habitual representan hombres y mujeres. En este punto se pretende establecer qué factores actúan en la frecuentación y estancia femenina como consecuencia de aspectos tales como las posibilidades de recreación y de ocio muy relacionados con las generaciones de pertenencia -pues el acudir y quedarse variará de acuerdo con la edad de las usuarias-; la sensación de incomodidad o de vulnerabilidad que se puede experimentar por permanecer sin compañía en el lugar; u otras razones ligadas a la adecuación física del espacio para la estancia, en cuanto a la disponibilidad y confort del mobiliario y de las restantes características ambientales (iluminación, vegetación, limpieza, etc.).

\section{ESPACIOS PÚBLICOS, GÉNERO Y DERECHOS CIUDADANOS}

Son muchos los factores que han incidido en la modificación de los espacios públicos abiertos en los que se desarrolla una parte de la vida ciudadana, entre los que cabe mencionar los cambios experimentados en la organización familiar y en los modos de vida; la percepción de inseguridad o la aparición de nuevos centros de diversión y ocio (Martínez, 2003; Rebughini, 2001; Sorkin, 1992). De hecho, el planeamiento en la ciudad contemporánea ha tendido a crear ámbitos que se basan más que en la interacción en la seguridad; más que en la heterogeneidad social y pluralidad de usos en la homogeneidad y el fomento del consumo (Fleury, 2007); y más que en la identidad e integración ciudadanas en su potencialidad como elementos útiles para la competitividad urbana y en su interés mercantil como imagen que simboliza los valores económicos imperantes. En esta dirección muchos sostienen, con 
argumentos más o menos similares, que no en vano la «noción de espacio público se puso de moda entre los planificadores, sobre todo a partir de las grandes iniciativas de reconversión urbana, como una forma de hacerlas apetecibles para la especulación, el turismo y las demandas institucionales en materia de legitimidad»(Delgado, 2011: 9). Pese a todo, no se puede negar que tales lugares de encuentro colectivo, de acceso libre y gratuito, son indispensables para el funcionamiento democrático (Fraser, 1990; Marston, 1990; Mitchell, 1995), al tiempo que la disponibilidad de espacios públicos de calidad y la posibilidad de su uso y disfrute constituyen un derecho ciudadano.

Muchos son, también, los que afirman que la existencia de este tipo de espacios propicia, mediante el desarrollo de relaciones de ayuda y de apoyo, tanto el sentimiento de pertenencia a una comunidad como una mejor calidad de vida. En ese sentido, todos tenemos derecho a disfrutar de lugares agradables, seguros, versátiles y accesibles que son, como se ha afirmado y con razón, más necesarios que los grandes equipamientos, inaccesibles pero susceptibles de ser inaugurados (Sánchez de Madariaga, 2004a: 95). Es decir, es imprescindible contemplar la presencia de este tipo de ámbitos para la vida cotidiana en la planificación y gestión urbanas, pues forman parte esencial de la sociabilidad básica de los individuos, familias o grupos de personas (Román, 1995; Jirón, 2007: 175) y son, pues, esenciales para facilitar la cohesión social.

Esto último supone que la ciudad y sus barrios deben contar con espacios que permitan y favorezcan, en lugar de limitar o restringir, la presencia y apropiación de los ciudadanos, aunque es cierto que no todos hacen uso de ellos con igual intensidad y de la misma manera. Existen diferencias en función de las características personales y sociales. No obstante conviene relacionar esas categorías. A este respecto, la edad puede explicar un acceso diferenciado a los lugares y la consecución de experiencias diversas; una dinámica que puede variar en función de las relaciones intergeneracionales (Hopkins y Pain, 2007). Del mismo modo el género tampoco es una categoría homogénea y sencilla, por lo que resulta necesario considerar su intersección con otras variables (Valentine, 2007). En todo caso, como categoría, el género ha quedado con frecuencia al margen del análisis teórico y empírico, oculta tras conceptos neutros como población, habitantes o familia (Falú, 2011: 132), aunque tiene un interés analítico estratégico cuando se aborda el tema de la presencia/participación de hombres y mujeres en los ámbitos públicos cotidianos. Por ello, su buen diseño y gestión -contemplando de forma equilibrada las necesidades de todos- constituiría una fórmula adecuada para lograr ciudades más equitativas. Desde la perspectiva de género, diseñar significa, como señala Segovia (2009: 156), hacerlo para la diversidad de personas y de situaciones colectivas, para la soledad y para el encuentro, para la intimidad y para la comunidad. Es de tal importancia la forma en la que se concreta la intervención, sea renovación o creación del espacio público, que puede reforzar o limitar las oportunidades para su apropiación (Harth, 2006). Tal es así que algunas investigaciones han puesto de relieve que «el diseño de los espacios públicos repercute más sobre la vida cotidiana de las mujeres que sobre la de los hombres debido a que éstas tienen una relación más estrecha con el entorno inmediato y realizan más actividades relacionadas con el trabajo reproductivo en este medio» (Ortiz, 2007: 23).

Parafraseando a Saskia Sassen, si para comprender la economía global es preciso considerar y hacer visible la dimensión de género (2003: 68), ésta sería también necesaria para 
entender el funcionamiento de la ciudad y de sus ámbitos de relación. Se impone así la necesidad de contribuir a examinar las diferencias entre hombres y mujeres en su uso y captar cuáles pueden ser las acciones que conducirían a una mayor equidad en la presencia y participación. Por tanto, no es extraño que Harvey afirme que el derecho a la ciudad es, en términos materiales, una quimera (2012: xvi, 16, 23), pues no todos tienen los mismos recursos simbólicos o materiales, entre ellos la mayoría de las mujeres, quienes desconocen sus derechos, viven restricciones y, en general, están en condiciones de mayor vulnerabilidad social. No sorprende, pues, que se siga afirmando que el ámbito público es, a pesar de las profundas transformaciones sociales, «masculino», dado que hay lugares y horarios donde la presencia de la mujer se sigue considerando socialmente inapropiada (Falú, 2011: 139). En cierto modo la existencia de apreciaciones de esta índole expresa cómo se reproducen las múltiples formas de asimetría que se forjan en la construcción cultural y social de los roles atribuidos a los sexos, lo que implica asignación de prioridades, pero también, de espacios (Muxí, 2006: 70). Doreen Massey (1994), en un trabajo ya clásico, afirmaba que los lugares y la opinión que de ellos tenemos se estructuran a partir del género, lo que supone reconocer las diversas formas en cómo se construye socialmente el género y cómo éste, a su vez, repercute en nuestras sociedades.

Con esta perspectiva de análisis se propone una reflexión sobre cómo actúa la administración al planificar este tipo de lugares y las limitaciones que éstos pudieran presentar para conseguir hacer factible el derecho de todos los ciudadanos a disfrutar del encuentro, de la estancia y de la sociabilidad que se deriva de la confluencia y participación colectivas. En este sentido, la dimensión de género constituye una manera de afrontar el análisis del uso y apropiación no como un asunto que atañe exclusivamente a las mujeres, sino como un enfoque que reclama la necesidad de que las políticas urbanas incorporen, como una cuestión central para avanzar hacia una mayor calidad de vida, la diversidad social (Saborido, 1999: 13). Es decir, el urbanismo y su concreción en los espacios públicos debe responder a las necesidades diferenciadas de acuerdo con el género, pero también con la edad, reconociendo y atendiendo las demandas de ancianos, personas con movilidad reducida (Penelas, 1998) o las que se derivan de las peculiares formas de juego de niños y niñas, así como de los familiares o personas que los acompañan. Esa adecuación a diferentes grupos, especialmente al femenino, pero también a otros de índole cultural o étnica, o a las personas de más edad, es lo que hace a la ciudad, según Jordi Borja, «un lugar conquistable por todos» (2003: 175). Una mirada como la que se plantea significa, en definitiva, considerar a aquellos colectivos que, como el de las mujeres, resultan por lo general más vulnerables y precisan por ello ámbitos que reúnan unos requisitos mínimos adaptados a sus necesidades. Esto último comporta dotar de operatividad a la noción «calidad de vida» que se vuelve muy imprecisa cuando se pasa de la teoría a la práctica o lo que es igual, del discurso institucional, a la implementación de políticas, planes y programas (Sánchez de Madariaga, 2004b: 102).

En definitiva, se trata de contribuir a hacer visibles las razones que pueden determinar las diferencias entre hombres y mujeres en el uso del espacio público y percibir las acciones que propiciarían una mayor presencia y participación de ambos. Se atenderá, pues, a las diferencias de uso y apropiación, partiendo de la consideración de que si no se consigue en estos ámbitos de la vida cotidiana un equilibrio de género, -que no sólo depende de 
una similar presencia de usuarios y usuarias sino, sobre todo, en la equidad o igualdad de oportunidades en la apropiación de estos espacios, así como una efectiva participación en su definición y gestión- será difícil superar las situaciones de desigualdad en otras esferas de interacción social.

\section{ANALOGÍAS Y DIFERENCIAS EN EL USO Y APROPIACIÓN DE LAS ALAMEDAS DE SEVI- LLA Y SANTA CRUZ}

\section{Las alamedas de Sevilla y Santa Cruz de Tenerife en su marco histórico y espacial}

Las alamedas de Hércules en Sevilla y del Duque de Santa Elena en Santa Cruz de Tenerife, aunque con génesis y desarrollos diferentes, se han elegido para este análisis porque son y han sido espacios destacados en la vida social de sus ciudades respectivas, al constituir lugares simbólicos y desde su inicio importantes puntos de reunión social. La construcción de la primera en 1574, tras la desecación definitiva de un antiguo brazo del río Guadalquivir, aportó el mayor espacio público intramuros de Sevilla, con una gran capacidad para acoger usos dispares y estamentos sociales variopintos. La realización más tardía de la segunda, en 1787, emplazada en la fachada marítima, conformó uno de los primeros lugares de esparcimiento de los grupos más acomodados y el único recinto público de Santa Cruz hasta 1838.

La evolución de las dos alamedas, desde la segunda mitad del siglo XIX hasta las primeras décadas del XX, muestra un desplazamiento de su relevancia social como epicentros de sus ciudades respectivas, motivado por las reformas urbanísticas que generaron nuevos espacios públicos de representación y de uso para las clases privilegiadas en otros ámbitos urbanos. En la actualidad constituyen, desde el punto de vista de la socialización, lugares de relación y encuentro tanto para los vecinos del área residencial cercana, como para los habitantes del resto de la ciudad, lo que se advierte en el notable volumen de personas que acude a ellas en su tiempo de ocio. Son ámbitos recientemente renovados, con diseños que tienen en común el conformar espacios abiertos de grandes dimensiones, peatonales, de fácil acceso interno y externo que se han acondicionado para el desarrollo de prácticas recreativas diversas (descanso, paseo, juego infantil...) y que, en consecuencia, podrían ser identificados como espacios propensos a la multifuncionalidad.

La reforma de la alameda de Hércules, culminada en 2008, significó, después de un proceso de deterioro físico y fijación de actividades marginales, su consolidación como lugar de ocio y comercio, pero también de creación y protesta. Paradójicamente, una parte notable de los nuevos vecinos en un entorno de creciente ascenso del precio del suelo, están atraídos por estos valores y son partícipes de la reactivación de movimientos sociales en la zona. Se demuestra así de nuevo la íntima relación entre la configuración física y la construcción funcional de este espacio respecto a la evolución de su entorno urbano (García, 2011; León, 2000; Álvarez et al,1982) cuya referencia más reciente es el desarrollo del proyecto URBAN San Luis Alameda a mitad de los años 90 y la remodelación de este ámbito en paralelo a cambios en las dinámicas socioeconómicas del norte del intramuros de la ciudad de Sevilla hasta la actualidad (Díaz, 2012; Barber et al, 2006; Cantero et al, 1999). Paradójicamente durante este periodo no fue variando tanto la configuración del espacio de la alameda, como su uso. Mantiene algunos muy reconocibles por grupos concretos de población, como el 
mercadillo dominical, como espacio de reivindicación o el propio de algunos locales nocturnos, pero llega a pasar por situaciones extremas como su utilización durante 2001 como gran aparcamiento improvisado.

La intervención de 2008 sí conlleva un intenso cambio formal, ya que la peatonalización de la totalidad del espacio ( $37 \mathrm{ha}$ ) recupera una imagen de conjunto inédita en los últimos decenios (Figura 1). Asimismo se replanta aunque con una eficiencia relativa parte del arbolado y se sitúan nuevos elementos dentro del paseo, configurando un espacio oblongo en el que el mobiliario y los espacios de uso -zonas de juego infantil, kioscos, etc.- se concentran en su flanco oriental (figura 1), marcando un espacio de paseo y otro de estancia a priori. Por su parte, la uniformidad del uso del color amarillo y de los materiales ha favorecido la homogeneización y cierta banalización de su imagen, mientras que se ha perdido un sustrato tan interesante como el albero original (García, Delgado y Ojeda, 2007).

Figura 1

ESQUEMA Y LOCALIZACIÓN DE LAALAMEDA DE HÉRCULES (SEVILLA)

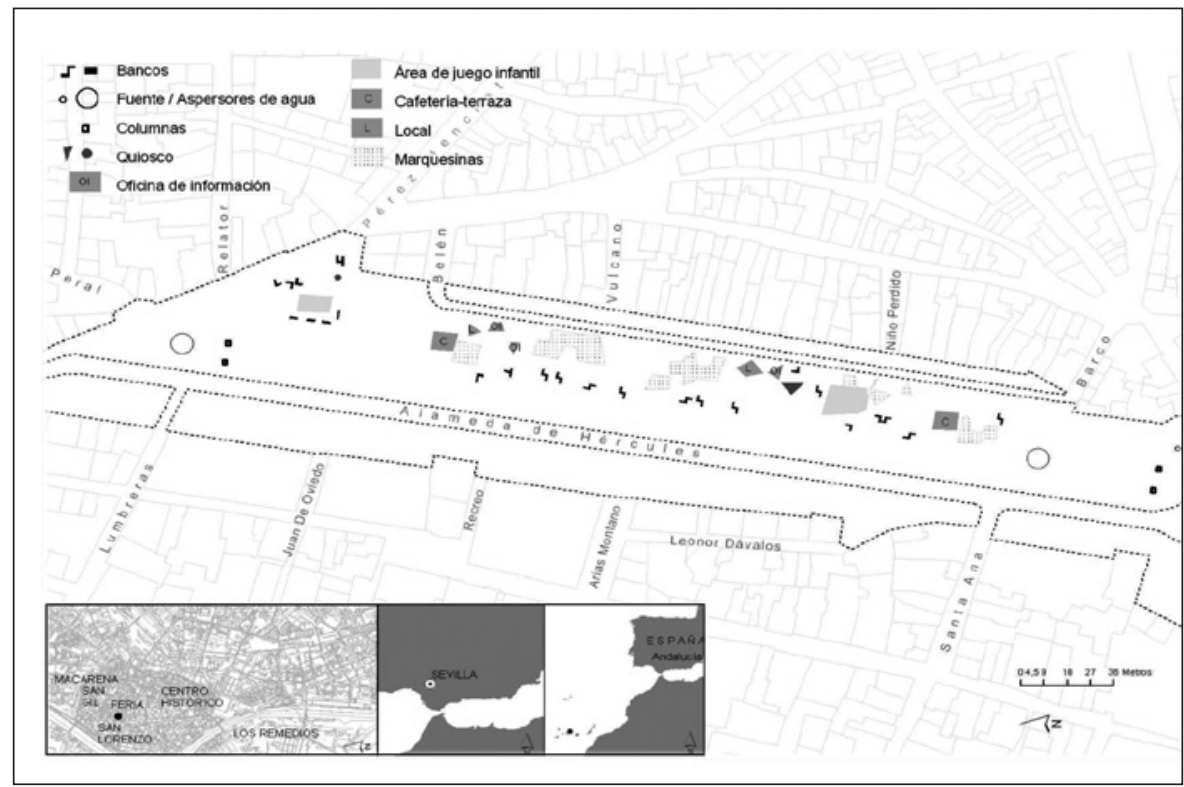

Fuente: Elaboración propia a partir de IDE.Sevilla, Gerencia de Urbanismo de Sevilla.

En Santa Cruz de Tenerife la remodelación de la alameda del Duque de Santa Elena, inaugurada en 2008, ha transformado, de igual modo, su fisonomía banalizándola al tiempo que desaparece como elemento singular para quedar integrada en un gran parque urbano (36 ha) que incluye otros dos lugares públicos lindantes: plazas de España y de La Candelaria (Figura 2). De hecho, se trata de una actuación que se inserta en un proyecto de mayor envergadura que se extiende hasta la zona portuaria y que supone la reordenación del Muelle de Enlace (Figura 2) y una parte sustancial del frente marítimo de Santa Cruz (Autoridad Portuaria, 2007: 16). Una intervención de este alcance persigue, entre otros fines, «la concen- 
tración de usos terciarios, el refuerzo y ampliación del área urbana destinada a los negocios, la rehabilitación de edificios degradados y la revalorización de operaciones de gran riesgo» (Casariego, 1999: 104), al tiempo que se crean nuevos espacios públicos (Díaz, García y Armas, 2010) y se renueva la actividad portuaria. Se trata, pues, de una estrategia general que ha sido aplicada por el gobierno de muchas ciudades en el intento de mejorar su competitividad urbana (Talesnik y Gutiérrez, 2002). Así pues, en la recuperación y embellecimiento de las alamedas de Sevilla y Santa Cruz se advierte una evidente preocupación por construir espacios representativos de los modelos urbanos de «vanguardia» donde el principal objetivo consiste en conseguir, sobre todo, diseños que revaloricen la imagen de la ciudad.

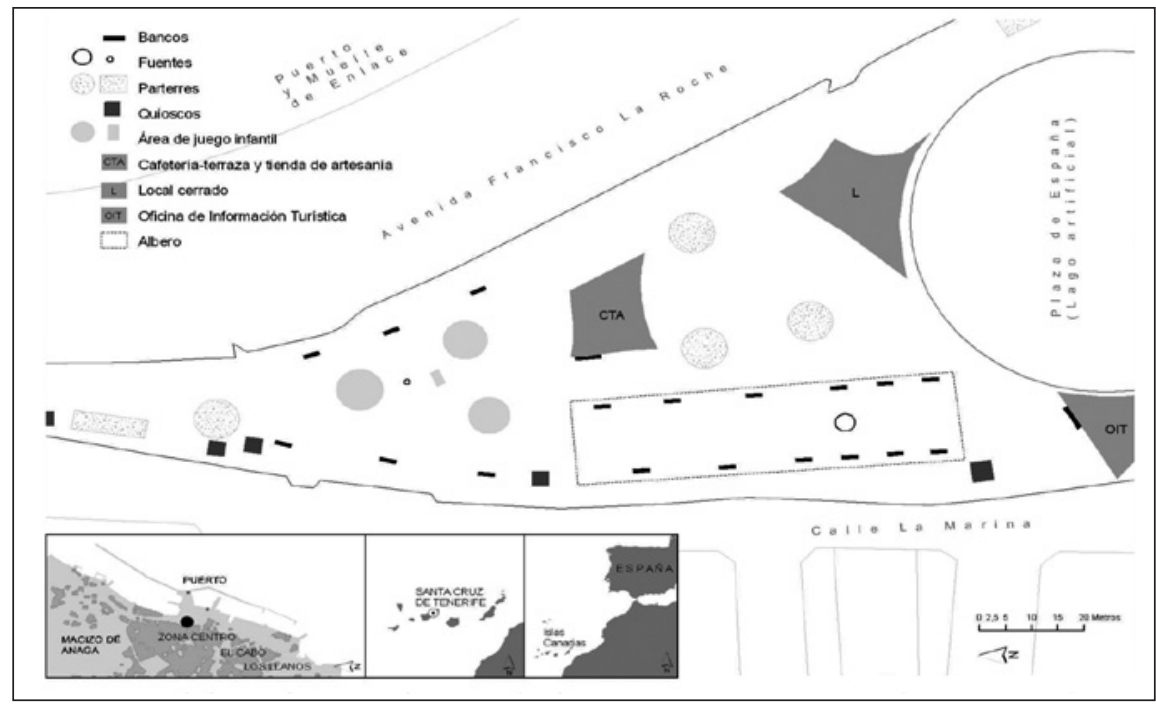

Fuente: Elaboración propia a partir de Cartográfica de Canarias (Grafcan), Gobierno de Canarias.

Con independencia de las peculiaridades de ambas, lo que sí parece haberse producido, tras los últimos cambios, es la reactivación de las dinámicas de uso, aunque éstas presentan diferencias en los grupos que las frecuentan y en los momentos en que lo hacen. Se estudian a continuación las prácticas sociales identificadas en las alamedas; mientras el análisis de los comportamientos en función del género se examinará de forma expresa en el siguiente apartado.

\section{Las alamedas: quiénes las usan, cuándo, cómo y con quién}

Antes de analizar las diferencias y similitudes que se observan en el uso de las dos alamedas (Tabla 1) es preciso hacer referencia al incremento del uso que éstas muestran tras sus últimas reformas. En el caso de la alameda de Hércules, de Sevilla, es muy evidente, tanto cuantitativamente como en lo relativo a la diversidad de usos y usuarios, a pesar de 
Tabla 1

SIMILITUDES Y DIFERENCIAS EN EL USO Y APROPIACIÓN DE LAS ALAMEDAS DE HÉRCULES Y DEL DUQUE DE SANTA ELENA

\begin{tabular}{|c|c|c|}
\hline $\begin{array}{l}\text { Características } \\
\text { básicas }\end{array}$ & $\begin{array}{l}\text { Alameda de Hércules } \\
\text { (Sevilla) }\end{array}$ & $\begin{array}{l}\text { Alameda del Duque de Santa Elena } \\
\text { (Santa Cruz de Tenerife) }\end{array}$ \\
\hline $\begin{array}{l}\text { Frecuentación } \\
\text { del espacio }\end{array}$ & $\begin{array}{l}\text { Amplio incremento numérico y } \\
\text { de diversidad de usuarios tras la } \\
\text { reforma }\end{array}$ & $\begin{array}{l}\text { Amplio incremento numérico y de } \\
\text { diversidad de usuarios tras la reforma }\end{array}$ \\
\hline Género & $\begin{array}{l}\text { Presencia similar } \\
\text { de ambos sexos }\end{array}$ & $\begin{array}{l}\text { Predominio de los varones } \\
\text { (debido a la destacada presencia de } \\
\text { jubilados) }\end{array}$ \\
\hline $\begin{array}{l}\text { Grupos etarios } \\
\text { mayoritarios }\end{array}$ & $\begin{array}{c}\text { Adultos } \\
\text { niños y jóvenes }\end{array}$ & $\begin{array}{c}\text { Adultos } \\
\text { ancianos } \\
\text { niños y jóvenes }\end{array}$ \\
\hline Frecuentación & $\begin{array}{c}\text { Tardes y } \\
\text { fines de semana }\end{array}$ & $\begin{array}{c}\text { Mañanas y } \\
\text { días laborables }\end{array}$ \\
\hline $\begin{array}{l}\text { Formas de estar } \\
\text { preferentes }\end{array}$ & $\begin{array}{c}\text { Paseo, conversación } \\
\text { y } \\
\text { juego y cuidado de los menores }\end{array}$ & $\begin{array}{c}\text { Descanso, conversación } \\
\text { y } \\
\text { juego y cuidado de los menores }\end{array}$ \\
\hline $\begin{array}{l}\text { Valoración de } \\
\text { las reformas }\end{array}$ & $\begin{array}{c}\text { El } 80 \% \text { de los entrevistados } \\
\text { valora positivamente la reforma } \\
\text { en particular la peatonalización, } \\
\text { la amplitud del recinto, así } \\
\text { como la diversidad de personas } \\
\text { y la vitalidad del espacio }\end{array}$ & $\begin{array}{l}\text { El } 80 \% \text { de los entrevistados expresa } \\
\text { descontento respecto al diseño y la } \\
\text { incomodidad e insuficiencia de los } \\
\text { bancos. En cambio se pronuncian } \\
\text { favorablemente en relación con la } \\
\text { ampliación de las áreas de juego } \\
\text { infantil y la animación del lugar. }\end{array}$ \\
\hline
\end{tabular}

Fuente: Elaboración propia a partir del trabajo de campo.

que este carácter más abierto haya podido favorecer la pérdida de peso de ciertas referencias como espacio contestatario o pretendidamente popular (García, 2011). Una tendencia similar se aprecia en la alameda del Duque de Santa Elena, de Santa Cruz de Tenerife, que refuerza su papel relacional tradicional con la introducción de áreas de juego infantil, así como sectores de cierta amplitud que resultan atractivos como espacios de reunión para los adolescentes y jóvenes.

Dicho esto, resulta de interés centrar el análisis del uso y los usuarios de estos espacios públicos considerando tres aspectos relevantes: los grupos etarios de mayor presencia; la frecuentación en las mañanas y tardes de las jornadas laborables y festivas y, por último, las formas de apropiación más características.

Con respecto a quiénes son los usuarios se aprecia en ambas alamedas que el conjunto más numeroso son los adultos, solos o acompañados con otras personas de igual edad o con niños a los que cuidan en las áreas de juego. En cambio, la importancia de los otros tramos 
de edad es muy distinta de una a otra. En la sevillana destacan con similar proporción niños y jóvenes, mientras que en la de Santa Cruz son los ancianos el grupo más cuantioso. Es difícil establecer de forma nítida qué determina estas diferencias generacionales, pero se puede apuntar el hecho de que el entorno residencial de la alameda sevillana ha experimentado un fuerte proceso de renovación morfológica y de sustitución social mucho más intenso y reciente que en el caso de Santa Cruz, lo que puede ser uno de los factores desencadenantes. Sin embargo, en Sevilla deben influir otros condicionantes ambientales y de percepción de ocio, pues durante las mañanas de los domingos se registra un incremento sustancial de las personas de mayor edad. Una tercera clave para interpretar la diferente presencia numérica de distintos grupos de edad es el ámbito de influencia de este espacio, de modo que mientras los perfiles más jóvenes relacionados con actividades de ocio provienen de distintos puntos de la ciudad, en general, los ancianos están más claramente ligados al vecindario próximo.

En una y otra alameda resulta fácil identificar áreas con mayor presencia de unos y otros grupos etarios, por ejemplo, las zonas de juego infantil son a la vez espacios de concentración de los adultos acompañantes, al igual que los jóvenes se reúnen, por lo general, en los bancos o ámbitos menos cercanos a estos sectores; mientras que en Santa Cruz, los ancianos prefieren los bancos del paseo central de la alameda en los que pueden encontrar áreas de sol y sombra. Por su parte, en Sevilla, se puede detectar con facilidad la concentración infantil en torno a las zonas de columpios y sobre todo en el extremo norte, el uso por los jóvenes de los bancos centrales, especialmente en horario vespertino, o la ubicación de los grupos gay que se congregan en las proximidades de sus locales de referencia. En cuanto al comportamiento espacial de indigentes o personas de apariencia marginal, que puede estar influido por sutiles señales de intolerancia, se observa una tendencia a agruparse en zonas resguardadas, pero también aparecen en los bancos centrales cerca de otros perfiles.

El análisis de los momentos de mayor afluencia revela la existencia de dispares pautas de frecuentación que parecen estar bastante condicionadas por la distinta ubicación en el tejido de la ciudad de cada una de las alamedas. Así, la de Santa Cruz emplazada en el área comercial y de negocios, y próxima a la zona portuaria, presenta más dinamismo y diversidad en los días laborables y en las mañanas coincidiendo con la mayor actividad del centro urbano a lo que se suma el aporte adicional que se deriva del movimiento portuario y de los «cruceristas» que arriban a la ciudad. Es quizá por esa animación y por otros factores ambientales (sol y temperaturas agradables) cuando los jubilados ocupan preferentemente el lugar. En la de Sevilla, en cambio, la afluencia es algo más intensa en las tardes y en los fines de semana, cuando se favorece la interrelación de su carácter de lugar de tránsito -entre el centro histórico, el sector septentrional de la ciudad y el cauce histórico- con una mayor dimensión como espacio de ocio. Tanto en la alameda de Sevilla como en la de Santa Cruz los más jóvenes -adolescentes en algunos casos- manifiestan patrones similares en sus preferencias horarias, dada su mayor afluencia nocturna y su escasa representación en las mañanas de los días festivos.

Por lo que respecta al cómo, es decir, a cuáles son las formas de apropiación del espacio, se advierte que las modalidades mayoritarias son las mismas pero con distinto peso en cada alameda. En la de Santa Cruz el descanso y la conversación son las actividades que realiza más de la mitad de los individuos registrados; en la de Sevilla, en cambio, destacan el paseo y la conversación. En ambas el juego y el cuidado de los niños siguen en importancia, lo que 
está justificado por la abundante presencia infantil, a la que hay que agregar en Santa Cruz los adolescentes que se desplazan con patines, monopatines y bicicletas por distintos sectores del lugar. Por último, la lectura aunque constituye una actividad minoritaria es clarificadora en términos cualitativos de las dinámicas de estancia y reconocimiento de estos lugares.

El patrón de uso habitual muestra una tendencia hacia la intensificación vespertina de actividades como el juego, mientras que el paseo, especialmente en las mañanas del domingo, es la manera de estar más característica de la alameda de Hércules, el descanso y la charla definen la de Santa Cruz. La desigual importancia que en ambos espacios adquieren esas principales modalidades de apropiación está relacionada con las peculiaridades en la configuración formal, el trazado y la dotación de mobiliario y otros elementos en cada alameda (figuras 1 y 2), especialmente en aquellos casos de mayor vinculación con sus usos, como bancos o zonas de juego. Pero, lejos de mostrar unas relaciones deterministas, las motivaciones de aquellos usuarios más definitorios de cada momento del día hacen variar el mapa de los usos.

El cómo y el cuándo están condicionados por quiénes. Es decir, se advierten paisajes cambiantes de usuarios y de apropiaciones en función de los días y horas en los que se lleva a cabo la observación. De hecho, un mismo espacio «en tiempos diferentes se convierte en lugares distintos (...) pues atrae a colectivos diferentes (...) y, por tanto, los usos que se hagan del mismo también lo serán» (González, 2008: 95). Estamos ante lo que Parke y Thrift (1980) llamaron «cronografía», para aludir al hecho, aplicable a todos los potenciales usuarios, de que las diferentes percepciones de hombres y mujeres que se experimentan en un mismo espacio son, con frecuencia, el resultado de distintos tiempos de uso en los que se producen aspectos estéticos y funcionales diferentes (citado por Páramo y Milena, 2007: 7). En esos ritmos cambiantes que se registran en la frecuentación y uso de las alamedas analizadas, así como en algunas de las disimilitudes comentadas hasta ahora, influye un factor adicional que no es otro que la dispar afluencia y formas de permanencia según género.

\section{Uso y apropiación según género: ser y estar en las alamedas de Sevilla y Santa Cruz}

El hecho de ser hombre o mujer es relevante desde el punto de vista del uso y apropiación que hacen unos y otras de los espacios públicos. El género, como construcción social, influye en las impresiones y sensaciones que se tienen cuando se acude y se usa este tipo de espacios. Es cierto, que las percepciones masculinas y femeninas son muchas veces distintas, pues la característica de pertenecer a uno u otro sexo constituye una cuestión cultural construida de forma colectiva, que condiciona las maneras de ser «ciudadano», en cuanto a sujeto de derechos, y de comportarse en el espacio público, en cuanto a la posibilidad de ejercerlos de forma plena. Además, en la complejidad de factores que intervienen a la hora de acudir y permanecer en un lugar, hay que resaltar que las circunstancias personales están muy marcadas por la generación de pertenencia. Y aunque este rasgo es común a los dos sexos adquiere especial importancia en el caso de las mujeres que, bajo el influjo de patrones educativos y sociales estereotipados, perciben y, en consecuencia, adoptan pautas específicas de comportamiento en relación con su realidad cotidiana y también, con respecto a las maneras de disfrutar en los espacios públicos (Páramo y Milena, 2007). Esto nos lleva a prestar atención -a falta de un análisis en profundidad de las opiniones y visiones de los diferentes usuarios 
según sus diversas realidades sociales y culturales-a las desiguales conductas observables de frecuentación y formas de estar que se registran en el espacio público en función de la edad.

En una primera aproximación se puede afirmar que las diferencias de género son notables entre las dos alamedas. Así uno de los rasgos definitorios de la alameda sevillana es la existencia de un equilibrio entre el número de hombres y el de mujeres (Tabla 2). Y lo que es más importante, que la distribución en el espacio de unos y otras, así como las actividades que realizan, no muestran patrones excluyentes. Esto queda de manifiesto en las formas más habituales de uso y apropiación, como son el paseo, la charla y el cuidado/juego de niños con ligeras variaciones porcentuales entre uno y otro sexo que no resultan especialmente significativas (Tabla 2).

Tabla 2

DISTRIBUCIÓN PORCENTUAL DE LOS USUARIOS, SEGÚN SEXO Y GRANDES GRUPOS DE EDAD

\begin{tabular}{|c|c|c|}
\hline Sexo y grupos de edad & Alameda de Hércules & $\begin{array}{c}\text { Alameda del Duque de } \\
\text { Santa Elena }\end{array}$ \\
\hline Mujeres & 50 & 41 \\
\hline Jóvenes & 17 & 7 \\
\hline Adultos & 28 & 27 \\
\hline Ancianos & 5 & 7 \\
\hline Varones & 50 & 59 \\
\hline Jóvenes & 13 & 13 \\
\hline Adultos & 29 & 27 \\
\hline Ancianos & 8 & 19 \\
\hline Total & 100 & 100 \\
\hline
\end{tabular}

Fuente: Elaboración propia a partir del trabajo de campo.

En relación con el primer aspecto, el relativo al equilibrio de usuarios de uno y otro sexo, parece estar vinculado en Sevilla a la importante afluencia de mujeres adultas (Figura 2), que en una proporción similar a la de los varones, acuden en las mañanas y en las tardes de los días laborables y festivos. Se trata, muchas veces, de grupos familiares, de forma que adquiere importancia la cantidad de parejas con o sin hijos, lo que puede ser, en parte, producto de la recuperación física y de los cambios socioeconómicos del entorno residencial de la alameda, pues, en otros lugares, se ha llegado a comprobar que ...«muchos barrios elitizados tienen una alta proporción de mujeres, hecho que ha supuesto, de alguna forma, la «feminización» de la ciudad, con una nueva presencia de mujeres y niños en los espacios públicos» (Bondi, 1998 citado por Ortiz, 2007: 15). Pero esta presencia femenina no reproduce una clara diferenciación de género en cuanto a los usos del espacio, siendo por ejemplo indistinto el lugar o el motivo de los juegos infantiles, o ampliamente repartida las tareas de cuidado de niños entre las parejas jóvenes. Sí cabría señalar algunos matices en relación a las personas de mayor edad, ya que aunque su presencia es porcentualmente muy 
similar y se pueden reconocer usos análogos, parece que las mujeres de este grupo de edad están más volcadas al cuidado de pequeños, sobre todo por las mañanas, lo que condiciona los datos recogidos en la tabla 3.

Por su parte, es preciso tener en cuenta que, como se ha dicho, la atracción como lugar de encuentro de la alameda de Hércules ha ido en incremento, superando en mucho a su entorno más próximo y diversificando perfiles (García, 2011), por lo que a esta relación entre cambios socioeconómicos y género del espacio, cabe añadir referencias culturales propias de un amplio porcentaje de sus usuarios habituales. Así, si en anteriores decenios la alameda de Hércules había sido un icono de emancipación ciudadana (Lees, 2004) o de movimientos contraculturales, la mayor afluencia de personas ha suavizado este rol, aunque siguen siendo observables comportamientos inclusivos, de tolerancia o de equidad en los usos del espacio.

Figura 2

ALAMEDA DE HÉRCULES (SEVILLA). MUJERES Y NIÑOS EN LA MAÑANA DOMINICAL
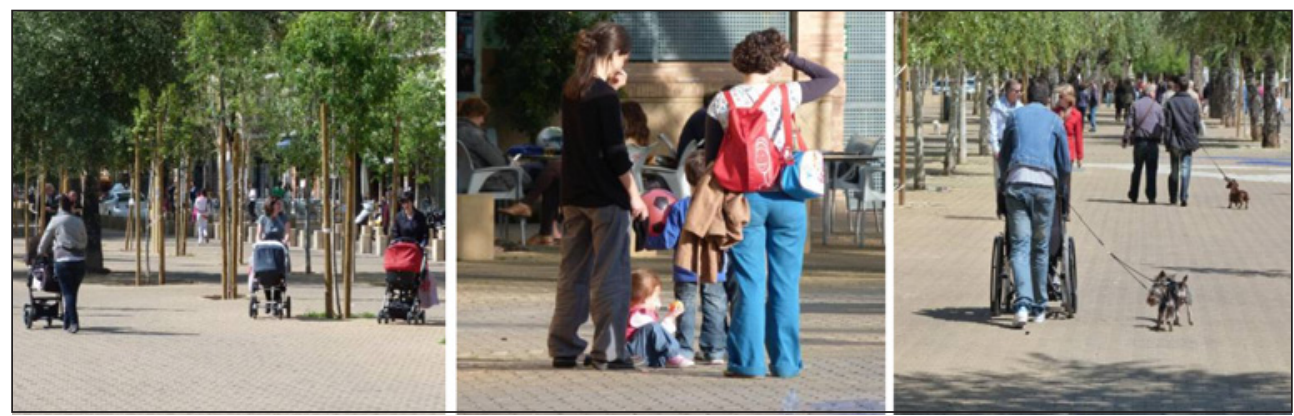

Fuente: Los autores.

La opinión de los entrevistados sobre las cualidades del espacio después de su reforma parece mayoritariamente positiva (80\%), y se valoran de modo especial, por un lado, cuestiones físicas como la peatonalización y la consecución de un recinto amplio para el uso peatonal y, por otro, la diversidad de personas y la animación y vitalidad que de ello se deriva, al confluir usuarios tradicionales y recientes, entre los cuales se destaca la incorporación de familias con niños $(40 \%)$ y gente de mayor poder adquisitivo (20\%). Paradójicamente las observaciones realizadas evidencian que si bien en la Alameda aparecen distintos perfiles de usuarios y motivaciones de uso, no llega a producirse una mezcla efectiva entre los distintos colectivos.

No parece que suceda exactamente lo mismo en la alameda de Santa Cruz, en la que predominan los varones en todas las observaciones realizadas (59 frente al 41\%). Se trata, no obstante, de una diferencia que responde a la destacada afluencia de jubilados, que acuden para sentarse al aire libre y charlar, sobre todo, por las mañanas (Figura 3). Es tal la importancia de este colectivo de varones de edad -su proporción representa una quinta parte del total de usuarios- que es el segundo en número después del grupo de adultos, superando al de los niños y también al de los jóvenes. La explicación de este hecho se encuentra, en parte, en el alto nivel de envejecimiento que, a pesar de los procesos de elitización registrados, presentan los ámbitos residenciales más o menos próximos a la alameda. Si nos quedáramos en este primer nivel de análisis se podría concluir que este espacio ofrece desde la perspec- 
tiva de género un cierto nivel de masculinización, lo que, debería ser matizado a través del análisis conjunto de género y generación. Tras el superior volumen de varones se oculta $-\mathrm{y}$ por tanto se desvirtúa- el alcance real que tienen las mujeres adultas en edad reproductiva. Éstas adquieren, al igual que en Sevilla, un peso similar o incluso levemente superior, en los diferentes turnos de observación, al de los varones de igual edad. Su presencia se percibe, en particular en las tardes y en los días festivos, acompañando y compartiendo juegos con niños de corta edad. De hecho parece que la dotación de sectores dedicados a ese específico uso ha propiciado, aunque quizá de una manera más difusa y menos evidente que en el caso sevillano, la afluencia de grupos familiares que por su carácter tienden a equilibrar la representación femenina.

Figura 3

ALAMEDA DEL DUQUE DE SANTA ELENA EN SANTA CRUZ DE TENERIFE. LA DIVERSA FRECUENTACIÓN EN LAS MAÑANAS DE LOS DÍAS LABORABLES

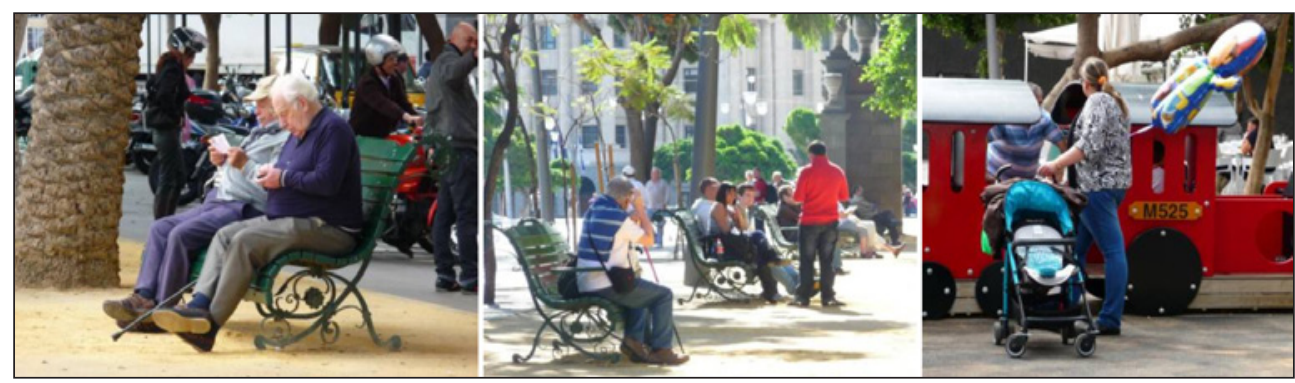

Fuente: Los autores.

Las diferencias están, por tanto, ocasionadas sobre todo por la escasez de las mujeres de edad que si no fuera por su papel de «cuidadoras» tendrían seguramente un peso inferior al $7 \%$ que registran -frente al $19 \%$ de los varones de igual grupo de edad-. Es infrecuente encontrarlas descansando o charlando como sí ocurre con sus congéneres masculinos, cuando lo normal sería lo contrario dado el reparto etario en estas edades donde ellas son mayoría. Las tareas reproductivas que asumen en sus hogares o contribuyendo como apoyo en el de sus hijos -custodia de nietos-, es una realidad cotidiana para muchas de ellas que, sin duda, limita o restringe sus posibilidades de reposo y recreación, cuya calidad se resiente al estar mediatizada por su responsabilidad en el cuidado de otros. De todos modos, no se pueden descartar tampoco otros aspectos vinculados con pautas arraigadas de comportamiento entre las que se encuentra la escasa satisfacción personal que algunas mujeres pueden experimentar al permanecer sin compañía en el lugar, algo que es muy usual, en cambio, entre ellos.

En el extremo opuesto de edad, las mujeres más jóvenes muestran también cierta subrepresentación, aunque ésta parece más el resultado de una afluencia adicional de pequeños grupos de jóvenes-adolescentes para la práctica con monopatines y bicicletas donde ellos son claramente mayoría; de hecho, en las tardes el reparto según género es más igualitario en estos tramos de edad.

El análisis de las prácticas de recreación es en la alameda de Santa Cruz, a diferencia de lo que se comprueba en Sevilla, un aspecto que presenta comportamientos globales distintos 
entre ambos sexos. Así, mientras que para las mujeres la principal actividad es la de cuidar y atender a los niños en las áreas de juego (Tabla 3) -con un peso similar al descanso seguido por la charla-, en el caso de los varones el cuidado es la forma a la que dedican su tiempo la menor proporción de ellos. Aquí, encontramos de nuevo, un sesgo de edad, un tema generacional ya planteado, pues la cifra de los varones mayores desplaza a un tercer lugar la manera de esparcimiento de los adultos que suelen estar presentes también -en especial en las tardes de los domingos-, con o sin sus parejas, en las zonas de juego al cuidado de los niños, aunque es también verdad que ellos se ocupan de estas actividades en menor medida que ellas.

Tabla 3

DISTRIBUCIÓN PORCENTUAL DE LAS MODALIDADES DEAPROPIACIÓN SEGÚN SEXO

\begin{tabular}{|c|c|c|c|c|}
\hline \multirow[b]{2}{*}{ Formas de apropiación } & \multicolumn{2}{|c|}{ Mujeres } & \multicolumn{2}{|c|}{ Varones } \\
\hline & $\begin{array}{l}\text { Alameda } \\
\text { de } \\
\text { Hércules }\end{array}$ & $\begin{array}{l}\text { Alameda del } \\
\text { Duque de } \\
\text { Santa Elena }\end{array}$ & $\begin{array}{l}\text { Alameda } \\
\text { de } \\
\text { Hércules }\end{array}$ & $\begin{array}{c}\text { Alameda del } \\
\text { Duque de } \\
\text { Santa Elena }\end{array}$ \\
\hline Descansar & 7,5 & 34,1 & 10,9 & 41,9 \\
\hline Charlar & 28,8 & 24,2 & 29,5 & 26,1 \\
\hline Pasear & 31,5 & 4,5 & 33,8 & 3,0 \\
\hline Cuidado y juego niños & 22,8 & 34,8 & 15,8 & 16,7 \\
\hline Otros & 9,4 & 2,4 & 10,0 & 12,3 \\
\hline Total & 100,0 & 100,0 & 100,0 & 100,0 \\
\hline
\end{tabular}

Fuente: Elaboración propia a partir del trabajo de campo.

En Santa Cruz, a diferencia de los resultados que se obtienen en Sevilla, se advierte cómo las reformas efectuadas en la alameda son, mayoritariamente, valoradas de forma negativa (80\%); expresando los usuarios un amplio desagrado hacia elementos centrales del diseño -como el lago artificial aledaño integrado en el conjunto reordenado y las luminarias-, así como el descontento que algunos manifiestan acerca de la incomodidad e insuficiencia de los bancos. Los que aportan opinión favorable, remiten a la ampliación de las áreas de juego para niños y a la mayor animación del espacio.

\section{CONCLUSIONES}

En relación con el impacto de la renovación y el diseño actual de las alamedas de Sevilla y Santa Cruz de Tenerife en la cantidad y características de los usuarios, así como respecto a las pautas de uso y apropiación según género y generación, es difícil aportar respuestas concluyentes, sin un conocimiento en profundidad de las razones de diversa índole de los que acuden y de los que dejan de hacerlo; habría pues que ahondar en la realidad social y cultural, así como en las percepciones de los sujetos que emplean las alamedas en su tiempo libre para cualificar y explicar las diferencias observadas. Aun así, partiendo de la validez metodológica de la observación, la descripción y el registro de lo que acontece en dichos espacios, se 
puede aventurar que las transformaciones que han dado la forma actual a las dos alamedas apuntan a ciertos tipos de usuarios, patrones de comportamiento y prácticas recreativas.

En lo que se refiere al efecto de la reordenación sobre los usuarios, se puede establecer una clara analogía entre los dos ámbitos estudiados de Sevilla y Santa Cruz con respecto al incremento de la frecuentación, así como a la diversificación de los usuarios entre los que adquieren un papel especial los grupos familiares. La recreación en un contexto familiar parece haberse visto favorecida por la introducción de zonas específicas de juego para niños de corta edad, así como por la ampliación de la superficie peatonal para el tránsito y el paseo. Estos aspectos inciden en la presencia de adultos de uno y otro sexo que muestran en las dos alamedas un peso similar. La incorporación de veladores y kioscos parece haber contribuido también al uso de estos espacios, en los que a los residentes se suman los visitantes. No obstante, se detectan todavía conflictos básicos entre necesidades y dotaciones. Así por ejemplo, en Santa Cruz las mujeres expresan cierto descontento con las áreas cercanas a los espacios de juego por su escaso número de asientos y zonas en sombra. En el caso de Sevilla en los últimos años se han producido conflictos entre el intento de eliminar asientos en estas zonas y la resistencia de sus usuarios habituales. Los kioscos y cafeterías son un recurso que cubre para algunos, esas carencias funcionales que repercuten en las personas acompañantes. Lo que, sin embargo, no representa una solución real ya que no todos pueden hacer igual uso, dada su índole privada. El diseño parece, pues, que propicia cierto tipo de usuarios con pautas de esparcimiento y apropiación homogéneas. La calidad de uso y la pluralidad de usuarios pueden, por tanto resentirse, en estos casos, por la búsqueda de un efecto de diseño, que a veces puede resultar resulta y contraproducente para la heterogeneidad social y multifuncionalidad de actividades.

En cuanto a la relación entre las prácticas sociales y las categorías de género y edad, se constata un sesgo notable en los usuarios de la alameda de Santa Cruz, no así en la sevillana. La presencia destacada en la primera de varones jubilados reduce el peso global de las mujeres, aunque en realidad son las de mayor edad las que están subrepresentadas. La combinación del nivel de envejecimiento del vecindario y la dedicación doméstica de la población femenina de mayor edad son dos aspectos, entre otros posibles, que explican tales diferencias generacionales y de género. En cualquier caso, sí parece que ellos suelen relacionarse con conocidos o amigos en mayor medida que ellas, que aparecen generalmente acompañando a hijos y nietos o incluso a personas mayores dependientes.

Si prestamos atención a las actividades o maneras de apropiación que se desarrollan en ambas alamedas, encontramos que las diferencias están más condicionadas por la edad que por el género. La división tradicional de tareas se advierte de forma nítida entre los mayores y en menor medida entre los adultos, en los que a pesar de los cambios sociales y culturales tendentes a la aproximación de comportamientos entre los dos sexos, sigue existiendo un sesgo por parte de ellas de mayor dedicación de su tiempo al contexto familiar y a las tareas vinculadas al acompañamiento y al cuidado.

En resumen, la reordenación de las alamedas de Sevilla y Santa Cruz parece haber ampliado las posibilidades de recreación en los sectores urbanos en los que se insertan; de igual forma se aprecia una diversificación de los usuarios, producida tanto por una mejora en las condiciones de uso, como por la aceptación de estos espacios públicos como lugares multifuncionales por distintos grupos de usuarios, incluyendo tanto a residentes cercanos, como a usuarios procedentes de otras zonas de la ciudad y visitantes Por último, aunque se 
incorporan dotaciones casi imprescindibles cuando el espacio público tiene unas mínimas dimensiones (áreas de juego infantil, asientos, vegetación...) y con ello se amplían las posibilidades de esparcimiento y de ocio, no es, en cambio, seguro que esas dotaciones cubran de forma suficiente y adecuada las diversas necesidades de los usuarios efectivos y de los potenciales usuarios cotidianos, es decir, de aquellos que residen en el entorno más o menos próximo. Esta carencia de calidad del diseño para la sociabilidad cotidiana es, en definitiva, producto de que en la transformación llevada a cabo ha prevalecido la preocupación y el criterio estético sobre el funcional; aspecto que padecen, eso sí, por igual hombres y mujeres.

\section{AGRADECIMIENTOS}

Agradecemos el trabajo del estudiante de Geografía de la Universidad de La Laguna, Juan Samuel García Hernández, por las encuestas realizadas en la alameda del Duque de Santa Elena y su contribución en el vaciado, así como las mejoras sugeridas por los revisores en una versión previa de este artículo.

\section{BIBLIOGRAFÍA}

ÁlVAREZ, L.; COLLANTES, A. y ZOIDO, F. (1982): «Plaza, Plaza Mayor y espacios de sociabilidad en Sevilla intramuros». Place et sociabilité en Europe et Amérique Latine, fasc. VII, 81-102.

AUTORIDAD PORTUARIA DE SANTA CRUZ DE TENERIFE (2007): Memoria de Información del Plan Especial del Puerto de Santa Cruz de Tenerife. En tramitación. Disponible en http://www.puertosdetenerife.org/Portal.aspx?p_ObjId=4647\&p_ PObjId $=4628 \&$ VerDet $=$ true

BARBER, S., FRENSEL, M. y ROMERO, M. J. (coord.) (2006): De cómo nació, creció y se resiste a ser comido. El gran pollo de la Alameda, una docena de años de lucha social en el barrio de la Alameda, Sevilla. Sevilla, Publigrupo.

BORJA, J. (2003): La ciudad conquistada, Madrid, Alianza Editorial.

CANTERO, P., ESCALERA, J., GARCÍA, R. y HERNÁNDEZ, M. (1999): La ciudad silenciada: vida social y Plan Urban en los barrios del casco antiguo de Sevilla. Sevilla, Ayuntamiento de Sevilla.

CASARIEGO, J., GUERRA, E., LEY, P. y PALOP, J. (1999): Waterfronts de nuevo: transformaciones en los frentes urbanos de agua. Las Palmas de Gran Canaria, Ayuntamiento de Las Palmas de Gran Canaria.

DELGADO, M. (2011): El espacio público como ideología. Catarata, Barcelona.

DÍAZ, I. (2012): «Intervención urbanística y disciplinamiento en los centros históricos. La creación de un espacio seguro en San Luis-Alameda (Sevilla)» en Actas del XI coloquio de Geografía Urbana: Las ciudades y el sistema urbano. Reflexiones en tiempos de crisis (Miramontes, A., Royé, D. y Villa, J. I.), Santiago de Compostela, MeuBook, 121-130.

DÍAZ RODRÍGUEZ, M.C., GARCÍA HERRERA, L.M. y ARMAS DÍAZ, A. (2010): «Puertos y espacios públicos renovados: el puerto de Santa Cruz de Tenerife» en Actas del XVIII Coloquio de Historia Canario-Americana (Padrón Morales, F.), Las Palmas de Gran Canaria, Cabildo Insular de Gran Canaria, 914-922. 
FALÚ, A. (2011): «Restricciones ciudadanas: las violencias de género en el espacio público». Pensamiento Iberoamericano, $\mathrm{n}^{\circ}$ 9. (Ejemplar dedicado a feminismo, género e igualdad), 127-146.

FLEURY, A. (2007): Les espaces publics dans les politiques métropolitaines. Réflexions au croisement de trois expériences: de Paris aux quartiers centraux de Berlin et Istambul. Tesis doctoral. Disponible en http://tel.archives-ouvertes.fr/tel-00259957/en/.

FRASER, N. (1990): «Rethinking the Public Sphere: A Contribution to Actually Existing Democracy». Social Text, vol. 25, n 26, 56-79.

GARCÍA, A. (2006): Vitalidad y crisis en los espacios públicos de Sevilla. Sevilla, Diputación de Sevilla.

GARCÍA, A. (2011): La calle a escena. El sistema de espacio público de Sevilla y su entorno metropolitano, retos y posibilidades Sevilla. Sevilla, Ayuntamiento de Sevilla.

GARCÍA, A., DELGADO, B. y OJEDA, J. (2007): «Paisajes simbólicos de la ciudad de Sevilla». Ería. Revista cuatrimestral de Geografía, no 73-74, 291-310.

GARCÍA-RAMÓN, M.D., ORTIZ, A. y PRATS, M. (2004): «Urban planning, gender and the use of public space in a peripherial neighbourhood of Barcelona». Cities, vol. 21, $\mathrm{n}^{\mathrm{o}}$ 3, 215-223.

GONZÁlEZ, E. (2008): Percepción y uso de espacios públicos madrileños. Tesis doctoral. Disponible en http://eprints.ucm.es/8115/.

HARTH, A. (2006): «Open Space and Gender - Gender-Sensitive Open-Space Planning». German Journal of Urban Studies, $\mathrm{n}^{\circ}$ 1. Disponible en http://www.difu.de/node/5961

HARVEY, D. (2012): Rebel Cities. From the Right to the City to the Urban Revolution. Londres, Verso.

HOPKINS, P. y PAIN, R. (2007): «Geographies of Age: Thinking Relationally». Area, vol. 29, no 3, 287-294.

JIRÓN, P. (2007): «Implicaciones de género en las experiencias de movilidad cotidiana urbana en Santiago de Chile». Revista Venezolana de Estudios de la Mujer, vol.12, no 29, 173-197.

LEES, L. (2004): The Emancipatory City? Paradoxes an Possibilities. Londres, Sage.

LEÓN, J. (2000): La Alameda de Hércules y el Centro Urbano de Sevilla; hacia un reequilibrio del Casco Antiguo. Sevilla, Universidad de Sevilla.

MARTÍNEZ, E. (2003): «La significación social de los espacios públicos». Mediterráneo económico, $\mathrm{n}^{\circ}$ 3, (Ejemplar dedicado a: Ciudades, arquitectura y espacio urbano / coord. por Horacio Capel Sáez), 115-130. Disponible en http://www.fundacioncajamar.es/mediterraneo/revista/me0307.pdf.

MARSTON, S. (1990): «Who are 'The People': Gender, Citizenship, and the Making of the American Nation». Environment \& Planning D: Society and Space, vol. 8, n 4, 449-458.

MASSEY, D. (1994): Space, Place and Gender. Cambridge, Polity Press.

MITCHELL, D. (1995): «The End of Public Space? People's Park, Definitions of the Public, and Democracy». Annals of the Association of American Geographers, vol. 85, $\mathrm{n}^{\circ}$ 1, 108-133.

MUXÍ, Z. (2006): «Ciudad próxima. Urbanismo sin género». Ingeniería y Territorio, $\mathrm{n}^{\circ}$ 75, 68-75. Disponible en http://www.ciccp.es/revistaIT/textos/pdf/09-Zaida\%20 Mux\%C3\%AD\%20Mart\%C3\%ADnez.pdf.

ORTIZ, A. (2004): «Ús i apropiació de la Via Júlia i la rambla del Raval de Barcelona desde una perspectiva de gènere». Documents d'Anàlisi Geografica, vol. 44, 89-108. 
ORTIZ, A. (2007): «Hacia una ciudad no sexista». Territorios, vol. 16-17, 11-28. Disponible en http://revistas.urosario.edu.co/index.php/territorios/article/view/838/758.

PÁRAMO, P. y MILENA, A. (2007): «Mujer en el espacio público a partir de su rol social». Revista Pre-Til, vol. 13, no 5, 8-28. Disponible en http://iaps.scix.net/data/contrib/att/ cbfd.content.09142.pdf.

PARAVICINI, U. (2003): «Public Spaces as a Contribution to Egalitarian Cities» en City and Gender. International Discourse on Gender, Urbanism and Archicture, (Terlinden, U.) vol.12, Springer, Wiesbaden, 57-80.

PENELAS, M. L. (1998): «Sobre la ciudad de las damas, la ciudadanía y la ciudad». Boletín $C F+S, \mathrm{n}^{\circ}$ 7. (Especial Mujer y ciudad). Disponible en http://habitat.aq.upm.es/boletin/ n7/ampen.html.

PRATS FERRET, M, BAYLINA, M. y ORTIZ, A. (2012): «Métodos cualitativos y perspectivas alternativas para el estudio de los espacios públicos urbanos» en Actas del XIII Coloquio Ibérico de Geografía. Respuestas de la Geografía Ibérica a la crisis actual (Royé, D. Aldrey, J. A. Valcárcel, M., Pazos, M. y Piñeira, M.J.), Santiago de Compostela, MeuBook, 754-764.

RAINERO, L. y RODIGOU, M. (2003): «Indicadores urbanos de género. Instrumentos para la gobernabilidad urbana» en $51^{\circ}$ Congreso Internacional de Americanistas. Ponencia presentada a la mesa Espacio y género en el campo y la ciudad Latinoamérica. Santiago de Chile. Disponible en http://www.redmujer.org.ar/articulos/art_09.pdf

REBUGHINI, P. (2001): Violenza e spazio urbano. Rapresentazioni e significati della violenza nella città contemporanea. Milán, Guierini Studio.

ROMÁN, M. (1995): «La reconstrucción del espacio cotidiano». Ciudades para un futuro sostenible. Disponible en http://habitat.aq.upm.es/select-sost/ad3.html.

SABORIDO, M. (1999): Ciudad y relaciones de género. Documento de Referencia DDR/5. CEPAL. Disponible en http://www.eclac.org/publicaciones/xml/5/4965/ddr5e.pdf.

SÁNCHEZ DE MADARIAGA, I. (2004a): Urbanismo con perspectiva de género. Instituto Andaluz de la Mujer. Junta de Andalucía.

SÁNCHEZ DE MADARIAGA, I. (2004b): «Infraestructuras para la vida cotidiana y calidad de vida.» Ciudades: Revista del Instituto Universitario de Urbanística de la Universidad de Valladolid, $\mathrm{n}^{\circ}$ 8, 101-133. Disponible en http://dialnet.unirioja.es/servlet/busquedadoc $? \mathrm{t}=$ Ines+Sanchez+de+Madariaga $\& \mathrm{db}=2 \& \mathrm{~m}=\& \mathrm{fi}=\& \mathrm{ff}=\& \mathrm{td}=$ todo $\& \mathrm{idi}=0$.

SASSEN, S. (2003): Contrageografías de la globalización. Género y ciudadanía en los circuitos transfronterizos. Madrid, Traficantes de sueños.

SEGOVIA, O. (2009): «Convivencia en la diversidad: una mirada de género al espacio público» en Mujeres en la ciudad. De violencias y derechos (Falú, A.). Red Mujer y Hábitat de América Latina. Ediciones Sur, Chile, 145-159.

SORKIN, M. (Ed.) (1992): The variations on a theme park: the new American city and the end of public space. Nueva York, Hill and Wang.

TALESNIK, D. y GUTIÉRREZ, A. (2002): «Transformaciones de frentes de agua: la forma urbana como producto estándar». Eure, vol. 28, n 84, Disponible en http://www.scielo. cl/scielo.php?script=sci_arttext\&pid=S0250-71612002008400002\&lng=es\&nrm=iso

VALENTINE, G. (2007): «Theorizing and Researching Intersectionality: A Challenge for Feminist Geography». The Professional Geographer, vol. 59, nº 1, 10-21. 
